\title{
The diagnostic value of the Short Physical Performance Battery for sarcopenia
}

\author{
Steven Phu ${ }^{1,2}$, Ben Kirk ${ }^{1,2}$, Ebrahim Bani Hassan ${ }^{1,2}$, Sara Vogrin ${ }^{1,2}$, Jesse Zanker ${ }^{1,2,3}$, Solange Bernardo ${ }^{1,3}$ and \\ Gustavo Duque ${ }^{1,2,3^{*}}$ (1)
}

\begin{abstract}
Background: Sarcopenia is defined as the age-related loss of muscle mass, strength, and physical performance. The original European Working Group on Sarcopenia in Older Persons (EWGSOP1) definition, and its revision (EWGSOP2), provide new cut-points and alternate measures for sarcopenia diagnosis. However, sarcopenia is rarely diagnosed in clinical settings owing to its labor-intensive diagnostic process. Given the Short Physical Performance Battery (SPPB) is a quick, easily administrable, and objective measure of muscle strength and physical performance, both of which are key components of sarcopenia, this study examined the diagnostic value of the SPPB for this muscle disease.

Methods: A cross-sectional analysis of 294 community-dwelling older persons ( $\geq 65$ years) was conducted. Appendicular lean body mass [(ALM) divided by height squared (ALM $\left.\left./ h^{2}\right)\right]$, muscle strength (handgrip/sit to stand), and physical performance [gait speed, timed up and go (TUG) and SPPB] were assessed using validated procedures, while participants were diagnosed with sarcopenia following the EWGSOP1 and EWGSOP2 criteria. Diagnostic ability of the SPPB independently and combined with ALM $/ h^{2}$ for sarcopenia was determined using area under the curve (AUC). Potential cut-points were identified, and sensitivity and specificity calculated.
\end{abstract}

Results: Prevalence of sarcopenia ranged from 4 to $16 \%$ depending on the definition. The SPPB demonstrated moderate (AUC $=0.644-0.770$ ) value in diagnosing sarcopenia, and a cut-point of $\leq 8$ points in SPPB performance resulted in high sensitivity (82-100\%) but low specificity (36-41\%) for diagnosing those with severe sarcopenia.

Conclusions: The SPPB displayed acceptable value in diagnosing older adults with severe sarcopenia. Moreover, the high sensitivity of the SPPB when using the cut-point of $\leq 8$ suggests it may be a favorable screening tool for sarcopenia in clinical settings where ALM measurements are not available.

Keywords: Sarcopenia, Diagnosis, Short physical performance battery, Ageing

\section{Background}

Sarcopenia, the progressive loss of muscle mass, strength and function, associates with falls, fractures, disability and mortality [1]. As a result of these adverse outcomes, sarcopenia was added to the International Classification

\footnotetext{
* Correspondence: gustavo.duque@unimelb.edu.au

${ }^{1}$ Australian Institute for Musculoskeletal Science (AIMSS), The University of Melbourne and Western Health, Sunshine Hospital, 176 Furlong Road, St Albans, VIC 3021, Australia

${ }^{2}$ Department of Medicine-Western Health, The University of Melbourne, 176 Furlong Road, St. Albans 3021, VIC, Australia

Full list of author information is available at the end of the article
}

of Diseases (ICD-10), allowing for its diagnosis and management in clinical practice [2]. Currently, several definitions exist for the diagnosis of sarcopenia, with each presenting their own diagnostic criteria. These include the European Working Group on Sarcopenia in Older People (EWGSOP1 and 2), [3, 4] Sarcopenia Definition and Outcomes Consortium (SDOC), [5] International Working Group on Sarcopenia (IWGS) [6] and the population specific Asian Working Group on Sarcopenia (AWGS) [7]. In regards to the EWGSOP definition, its most recent update (EWGSOP2) [4] proposed new cut-

(c) The Author(s). 2020 Open Access This article is licensed under a Creative Commons Attribution 4.0 International License, which permits use, sharing, adaptation, distribution and reproduction in any medium or format, as long as you give appropriate credit to the original author(s) and the source, provide a link to the Creative Commons licence, and indicate if changes were made. The images or other third party material in this article are included in the article's Creative Commons licence, unless indicated otherwise in a credit line to the material. If material is not included in the article's Creative Commons licence and your intended use is not permitted by statutory regulation or exceeds the permitted use, you will need to obtain permission directly from the copyright holder. To view a copy of this licence, visit http://creativecommons.org/licenses/by/4.0/ The Creative Commons Public Domain Dedication waiver (http://creativecommons.org/publicdomain/zero/1.0/) applies to the data made available in this article, unless otherwise stated in a credit line to the data. 
points and a screening process beginning with the use of the SARC-F [8] for case finding. With these new cutpoints and alternate assessments, wide variations in prevalence from 5 to $26 \%$ have been reported, [9] which hinders the diagnosis and management of older adults at risk.

Diagnosing sarcopenia involves assessment of muscle mass, strength, and/or physical performance. In the EWGSOP definitions, dual x-ray absorptiometry (DXA) is recommended for the assessment of appendicular lean mass (ALM), corrected for height squared $\left(h^{2}\right)$. Meanwhile, handgrip strength and gait speed are favored for assessment of muscle strength and physical performance, respectively. However, substitute measures are also proposed including the sit to stand test (alternate for handgrip strength), the timed up and go (TUG) test, and the Short Physical Performance Battery (SPPB) as alternatives for gait speed.

Of these measures, the SPPB assesses muscle strength, balance and mobility via five timed components, all of which are quick, easy to administer, and don't require any specialized equipment [10]. As such, we sought to examine the diagnostic value of the SPPB for sarcopenia. This was carried out by first identifying the prevalence of sarcopenia using EWGSOP1 and EWGSOP2 definitions. Upon doing so, the diagnostic value of the SPPB in characterizing individuals with sarcopenia will be evaluated. Finally, cut-points for sarcopenia using the SPPB score will be identified and compared to those currently recommended.

\section{Methods}

This was a cross-sectional study of 294 communitydwelling older adults based in Melbourne, Australia. Participants attending the falls and fractures clinic for clinical assessment between 2016 and 19 were included. Participants fulfilled the following criteria: aged over 65 years with a history of falls or at risk of falls, ability to mobilize independently with or without the use of gait aids, and no history of cognitive impairment. This study was approved by the Western Health local Human Ethics Research Committee (DB2017.13 and QA2018.80-46,205).

\section{Assessments}

Participants undertook a comprehensive assessment which included the identification of risk for falls and fractures. Falls (in the past year) and fracture (in the past 5 years) history were determined by interview. Assessments of physical performance and balance, as well as body composition to determine ALM $/ \mathrm{h}^{2}$ using DXA (Hologic Inc., Bedford, MA, USA) were also performed. Participant reported fear of falling was determined using the Falls Efficacy Scale - International [11].

Physical performance measures conducted on participants included the assessment of height and weight for calculation of body mass index (BMI), handgrip strength, gait speed, TUG, and SPPB. Handgrip strength was assessed using a Jamar hydraulic dynamometer (Sammons Preston Inc.) with the participant seated with their arm resting on chair arms. Participants were instructed to squeeze the dynamometer at their maximal effort, with the test performed 3 times on each side and $30 \mathrm{~s}$ rest provided between each trial. The best score out of the 3 trials was recorded.

To assess gait speed, we used the GAIT Rite ${ }^{\circ}$ (CIR Systems Inc., Havertown, PA) instrumented walkway system $(580 \mathrm{~cm} \times 89 \mathrm{~cm} \times 0.625 \mathrm{~cm}$, sample rate $=120 \mathrm{~Hz})$. The GAIT Rite mat was used to record spatiotemporal data. It was positioned along a straight section of the walkway and participants were instructed to walk at their normal speed. Three trials were provided with the best gait speed recorded. Gait aids were used a necessary.

To assess TUG performance, we used a 3-m course [12]. Participants began in a seated position and were instructed to stand, walk $3 \mathrm{~m}$ to a marked area, then return to the starting seated position. The TUG test was performed twice at the participants normal speed with the best time of completion recorded. A gait aid was allowed for use as needed.

The SPPB is an assessment which includes 5 tests for lower limb function, including balance, strength, and mobility [10]. Balance assessments were composed of 3 parts which progressed in difficulty (feet together stand, semi tandem and full tandem stand). The aim of the balance tests was to stand for $10 \mathrm{~s}$ unaided, with the test progressing in difficulty after successful completion. Gait speed was assessed as previously described (using GAIT Rite assessment results). The 5 times sit to stand test was performed with the participant starting in the seated position. After confirming ability to perform 1 sit to stand action, participants were then instructed to stand and sit 5 times as quickly as possible, ensuring feet were flat on the floor. Scores were allocated according to performance, with an overall maximum score of 12 .

\section{Classifying sarcopenia}

Sarcopenia status was classified based on the EWGSOP1 and EWGSOP2 definitions. This study focused on those who were classified as having severe sarcopenia and therefore fulfilled the 3 criteria for muscle strength, $\mathrm{ALM} / \mathrm{h}^{2}$ and physical performance. Participants were classified as severely sarcopenic according to 7 different sarcopenia criteria. Using the EWGSOP1 definition, severe sarcopenia was defined as those with low $A L M / h^{2}$, gait speed and handgrip strength. For the EWGSOP2 definition, 6 severe sarcopenia groups were created based on fulfillment of cut-points for muscle strength (handgrip strength or sit to stand test), $\mathrm{ALM} / \mathrm{h}^{2}$ and physical performance (low gait speed, SPPB score or increased TUG time). 


\section{Statistical analysis}

Baseline characteristics for participants are presented as a median (IQR) or frequency (\%). The diagnostic value of the SPPB was assessed by calculating the area under the receiver operating characteristic (ROC) curve separately for each sarcopenia definition. This was repeated with the addition of $\mathrm{ALM} / \mathrm{h}^{2}$ to the model. Area under the ROC curve (AUC) of 0.7-0.8 was considered to be acceptable, with $0.8-0.9$ excellent and $>0.9$ outstanding [13]. Optimal cut-points for the SPPB in diagnosing sarcopenia were defined by 3 different methods: maximizing the product of sensitivity and specificity (Liu), [14] maximizing their sum (Youden) [15] and cut-point on the ROC curve closest to perfect sensitivity and specificity (Nearest 0,1) [16]. All analyses were performed using Stata 15.1 (StataCorp. 2017. Stata Statistical Software: Release 15. College Station, TX: StataCorp LLC).

\section{Results}

\section{Baseline characteristics}

A total of 294 participants (77.2\% women) with a median age of 78 years (IQR 73, 83) were included in this analysis (Table 1). Sarcopenia prevalence ranged from 4 to $16 \%$ depending on the criteria employed (Table 2). EWGSOP1 and 2 definitions based on handgrip strength, ALM $/ \mathrm{h}^{2}$ and gait speed showed similar prevalence of sarcopenia at 12 and 8\%, respectively. Highest prevalence of sarcopenia was evident when using the sit to stand, ALM $/ \mathrm{h}^{2}$ and SPPB or gait speed criteria at $16 \%$, with the lowest prevalence observed when diagnosing sarcopenia by handgrip strength, ALM $/ \mathrm{h}^{2}$ and TUG (4\%). Number of falls, performance in SPPB and gait speed were similar between all sarcopenia categories except those diagnosed using TUG for physical performance. Handgrip strength varied

Table 1 Baseline characteristics of participants $(n=294)$

\begin{tabular}{ll}
\hline & Median (IQR) \\
\hline Age (yrs) & $78.2(73,83)$ \\
Sex (women), n (\%) & $227(77.2 \%)$ \\
Weight $(\mathrm{kg})$ & $70(59,80.8)$ \\
Height (cm) & $157(152,164)$ \\
Body Mass Index $\left(\mathrm{kg} / \mathrm{m}^{2}\right)$ & $28.04(23.92,31.45)$ \\
ALM/h ${ }^{2}$ & $6.33(5.72,7.22)$ \\
Falls in past year & $2(1,3)$ \\
Fractures & $1(1,1)$ \\
Handgrip strength (kg) & $21(16,28)$ \\
SPPB total score & $7(5,9)$ \\
Timed Up and Go (s) & $16.14(11.63,21.50)$ \\
Gait Speed (m/s) & $0.70(0.53,0.92)$ \\
FES-I & $34(24,45)$ \\
\hline
\end{tabular}

$A L M / h^{2}$ Appendicular Lean Mass corrected for height squared, SPPB Short Physical Performance Battery, FES-I Falls Efficacy Scale - International between groups with those using the sit to stand test to diagnose low muscle strength exhibiting the highest handgrip strength. Prevalence for each sarcopenia group and baseline characteristics of participants in each sarcopenia category can be found in Table 2 .

\section{Diagnostic value of SPPB}

Use of the SPPB resulted in moderate diagnostic value for sarcopenia for all EWGSOP definitions with AUC ranging from 0.644 to 0.770 , as shown in Table 3 . The lowest AUC occurred when using the SPPB to diagnose sarcopenia by the recommended EWGSOP2 criteria of low handgrip strength, $\mathrm{ALM} / \mathrm{h}^{2}$ and gait speed (AUC = 0.644). Highest AUC for use of the SPPB was found when diagnosing EWGSOP2 sarcopenia according to the alternate measures of sit to stand, $\mathrm{ALM} / \mathrm{h}^{2}$ and TUG $(\mathrm{AUC}=0.770)$.

\section{Diagnostic value of SPPB combined with ALM $/ \mathrm{h}^{2}$}

When combining the SPPB with $\mathrm{ALM} / \mathrm{h}^{2}$, there was a significant increase in the diagnostic capability. AUC for both the EWGSOP1 and 2 was increased, with AUC in an 'excellent' range for all groups, between 0.846 and 0.897 . The lowest AUC was evident using the EWGSOP1 criteria for severe sarcopenia (AUC $=0.846$ ), with AUC observed in the EWGSOP2 criteria of sit to stand, ALM $/ \mathrm{h}^{2}$ and TUG performance $(A U C=0.897)$. An overview of the AUC for use of the SPPB combined with $\mathrm{ALM} / \mathrm{h}^{2}$ in diagnosing each severe sarcopenia group can be found in Table 3.

\section{Potential SPPB cut-points for diagnosing sarcopenia}

Optimal cut-points for use of the SPPB alone to diagnose sarcopenia varied depending on method and ranged from 4 to 8 points. All cut-points resulted in either high sensitivity and low specificity or vice versa (Table 4). The currently recommended cut-point of $\leq 8$ produced high sensitivity (82-100\%) but low specificity (36-41\%) for all sarcopenia definitions. Most commonly observed optimal cut-points in SPPB performance were 5 and 6, which resulted in sensitivity and specificity between 60 and $75 \%$.

\section{Discussion}

We examined the diagnostic value of the SPPB alone and in combination with $\mathrm{ALM} / \mathrm{h}^{2}$ in diagnosing sarcopenia in community-dwelling older adults. We characterized the sensitivity and specificity of currently recommended cut-points, in addition to new cut-points based on our study population, and found two main findings. Firstly, the SPPB alone resulted in moderate diagnostic value but increased to an excellent level when combined with DXA measured ALM $/ \mathrm{h}^{2}$. This was regardless of which definition (EWGSOP1 or EWGSOP2) was employed. Secondly, the currently recommended SPPB cut-point of $\leq 8$ showed high sensitivity for 
Table 2 Characteristics of participants according to sarcopenia groups

\begin{tabular}{|c|c|c|c|c|c|c|c|}
\hline & \multirow{2}{*}{$\begin{array}{l}\text { EWGSOP1 } \\
\text { ALM/h2 }+H G+ \\
\text { GS }\end{array}$} & \multicolumn{6}{|l|}{ EWGSOP2 } \\
\hline & & $\begin{array}{l}\mathrm{HG}+\mathrm{ALM} / \mathrm{h}^{2}+ \\
\mathrm{GS}\end{array}$ & $\begin{array}{l}\mathrm{HG}+\mathrm{ALM} / \mathrm{h}^{2}+ \\
\text { TUG }\end{array}$ & $\begin{array}{l}\mathrm{HG}+\mathrm{ALM} / \mathrm{h}^{2}+ \\
\mathrm{SPPB}\end{array}$ & $\begin{array}{l}\text { STS }+A L M / \\
h^{2}+G S\end{array}$ & $\begin{array}{l}\text { STS }+ \text { ALM } / h^{2}+ \\
\text { TUG }\end{array}$ & $\begin{array}{l}\text { STS }+A L M / h^{2}+ \\
\text { SPPB }\end{array}$ \\
\hline Prevalence & $36(12 \%)$ & $22(8 \%)$ & $12(4 \%)$ & $19(7 \%)$ & $46(16 \%)$ & $24(8 \%)$ & $47(16 \%)$ \\
\hline Age (yrs) & $81.5(76,84)$ & $82.5(76,84)$ & $83(78.5,84.5)$ & $82(76,85)$ & $80(75,84)$ & $80.5(76,84)$ & $81(76,85)$ \\
\hline $\begin{array}{l}\text { Sex (women), n } \\
(\%)\end{array}$ & 22 (61\%) & $14(64 \%)$ & $7(58 \%)$ & $12(63 \%)$ & $32(70 \%)$ & $16(67 \%)$ & $32(68 \%)$ \\
\hline Weight (kg) & $57.3(50.6,67.6)$ & $55.3(50,66)$ & $56.7(50.1,65.7)$ & $55.2(50,66)$ & $57.6(51,64.1)$ & $56.3(50.2,63.5)$ & $56(50,64)$ \\
\hline Height (cm) & $155(151,163)$ & $154(149,163)$ & $156(151,163)$ & $155(152,163)$ & $156(151,163)$ & $157(150,165)$ & $157(152,163)$ \\
\hline Body Mass Index & $\begin{array}{l}23.79(21.83, \\
26.19)\end{array}$ & $\begin{array}{l}23.41(20.81 \\
26.01)\end{array}$ & $\begin{array}{l}23.39(21.44 \\
25.61)\end{array}$ & $\begin{array}{l}22.97(20.28, \\
25.20)\end{array}$ & $\begin{array}{l}23.26(21.17 \\
26.01)\end{array}$ & $\begin{array}{l}22.49(21.45 \\
24.64)\end{array}$ & $\begin{array}{l}23.23(20.81 \\
25.20)\end{array}$ \\
\hline $\mathrm{ALM} / \mathrm{h}^{2}$ & $5.42(5.12,5.92)$ & $5.40(5.12,5.67)$ & $5.38(5.04,5.58)$ & $5.26(5.04,5.67)$ & $5.22(5.00,5.67)$ & $5.23(5.02,5.58)$ & $5.23(5.03,5.83)$ \\
\hline Falls in past year & $2(1,2)$ & $2(1,2)$ & $2(1,3.5)$ & $2(1,3)$ & $2(1,2)$ & $2(1,2.5)$ & $2(1,3)$ \\
\hline $\begin{array}{l}\text { Handgrip strength } \\
(\mathrm{kg})\end{array}$ & $16(12,20)$ & $13.5(11,17)$ & $13.5(12,19)$ & $12(11,18)$ & $20(15,24)$ & $19.5(15,22)$ & $20(14,24)$ \\
\hline SPPB score & $5.5(4.5,7.0)$ & $6(4,8)$ & $4(2.5,6.5)$ & $5(4,7)$ & $5.5(4,7)$ & $4.5(4,6)$ & $6(4,7)$ \\
\hline $\begin{array}{l}\text { Timed Up and Go } \\
\text { (s) }\end{array}$ & $\begin{array}{l}19.31(16.71 \\
24.39)\end{array}$ & $\begin{array}{l}19.50(16.71 \\
24.39)\end{array}$ & $\begin{array}{l}25.09(22.27 \\
33.94)\end{array}$ & $\begin{array}{l}19.77(16.81 \\
25.80)\end{array}$ & $\begin{array}{l}19.46(16.71 \\
24.39)\end{array}$ & $\begin{array}{l}25.09(21.62, \\
29.92)\end{array}$ & $\begin{array}{l}19.23(15.42, \\
24.39)\end{array}$ \\
\hline Gait Speed $(\mathrm{m} / \mathrm{s})$ & $0.54(0.39,0.69)$ & $0.51(0.38,0.66)$ & $0.38(0.26,0.61)$ & $0.50(0.38,0.65)$ & $0.56(0.40,0.70)$ & $0.46(0.33,0.62)$ & $0.56(0.40,0.71)$ \\
\hline FES-I & $36(25.5,48.5)$ & $36.5(29,52)$ & $50(29,59.5)$ & $38(29,57)$ & $37(26,48)$ & $43.5(26.5,52.5)$ & $36(25,48)$ \\
\hline
\end{tabular}

ALM $/ h^{2}$ Appendicular Lean Mass corrected for height squared, GS Gait speed, HG Handgrip strength, SPPB Short Physical Performance Battery, TUG Timed up and go, FES-I Falls Efficacy Scale - International

sarcopenia, however, a more conservative cut-point of $\leq 6$ provided the greatest specificity. It therefore appears that the SPPB represents an objective screening tool to diagnose those at risk of sarcopenia.

The SPPB has been used to monitor physical performance in both healthy community-dwelling older adults as well as hospitalized patients [17-20]. This measure is advantageous in that minimal equipment is required to perform the test, and objective data is provided on muscle strength, gait speed and standing balance. In addition, specific components of the SPPB can independently predict the risk of declines in activities of daily living, [21] falls, [22] hospitalization [23] and mortality [23]. Furthermore,

Table 3 Area under curve (AUC) for use of Short Physical Performance Battery (SPPB) alone, or in combination with Appendicular Lean Mass corrected for height $\left(A L M / h^{2}\right)$

\begin{tabular}{llll}
\hline Definition & Criteria & AUC & AUC \\
& & SPPB & SPPB $+\mathrm{ALM} / \mathrm{h}^{2}$ \\
\hline EWGSOP1 & $\mathrm{ALM} / \mathrm{h}^{2}+\mathrm{GS}+\mathrm{HG}$ & 0.678 & 0.846 \\
EWGSOP2 & $\mathrm{HG}+\mathrm{ALM} / \mathrm{h}^{2}+\mathrm{GS}$ & 0.644 & 0.849 \\
& $\mathrm{HG}+\mathrm{ALM} / \mathrm{h}^{2}+\mathrm{TUG}$ & 0.767 & 0.870 \\
& $\mathrm{HG}+\mathrm{ALM} / \mathrm{h}^{2}+\mathrm{SPPB}$ & 0.715 & 0.873 \\
& $\mathrm{STS}+\mathrm{ALM} / \mathrm{h}^{2}+\mathrm{GS}$ & 0.701 & 0.893 \\
& $\mathrm{STS}+\mathrm{ALM} / \mathrm{h}^{2}+\mathrm{TUG}$ & 0.770 & 0.897 \\
& $\mathrm{STS}+\mathrm{ALM} / \mathrm{h}^{2}+\mathrm{SPPB}$ & 0.703 & 0.889
\end{tabular}

GS Gait speed, HG Handgrip strength, SPPB Short Physical Performance Battery, TUG Timed up and go the SPPB can be used as a sensitive monitoring tool for lower limb physical performance with a change of 1 point considered clinical significant [24]. These factors place the SPPB as an ideal tool for the diagnosis of sarcopenia in clinical settings and subsequent monitoring of resistance exercise (RE) interventions which are, at present, the most efficacious treatment for this muscle disease [25]. While other tools such as handgrip strength are reliable, easy to administer and a good marker of whole-body strength, they are poor responders to RE interventions [26]. Thus, the strength component of the SPPB should be assessed by sit to stand time, which is a reliable marker of lower limb strength and is sensitive to change [4].

Our findings show the SPPB displayed acceptable diagnostic value for sarcopenia. When employing the most common cut-points of $\leq 6$, sensitivity and specificity was 65 and 63\%, respectively. This cut-point has been associated with more than 3-fold likelihood of recurrent falls for both men and women, [27] one of the primary adverse outcomes of sarcopenia. Indeed, those diagnosed as sarcopenic (using the EWGSOP1) reported a 3-fold increased risk of falls compared to non-sarcopenic adults [28]. When increasing this cut-point to $\leq 8$, the sensitivity and specificity increased to 100 and $41 \%$, respectively. In line with this, this is the cut-point recommended for poor physical performance by the EWGSOP criteria. Despite the excellent sensitivity, the poor specificity of this cutpoint indicates that the SPPB cannot be solely used for the diagnosis of sarcopenia. 
Table 4 Sensitivity and specificity for cut-points for sarcopenia based on definition

\begin{tabular}{|c|c|c|c|c|c|}
\hline Definition & Criteria & Method & Cut-point $(\leq)$ & Sensitivity $(95 \% \mathrm{Cl})$ & Specificity $(95 \%$ Cl) \\
\hline \multirow[t]{3}{*}{ EWGSOP1 } & \multirow[t]{3}{*}{$\mathrm{ALM} / \mathrm{h}^{2}+\mathrm{GS}+\mathrm{HG}$} & Liu, Youden & 7 & $81 \%(64,92)$ & $48 \%(41,54)$ \\
\hline & & Nearest $(0,1)$ & 6 & $61 \%(44,77)$ & $62 \%(55,68)$ \\
\hline & & Current literature & 8 & $89 \%(74,97)$ & $38 \%(32,44)$ \\
\hline \multirow[t]{18}{*}{ EWGSOP2 } & \multirow[t]{3}{*}{$\mathrm{HG}+\mathrm{ALM} / \mathrm{h}^{2}+\mathrm{GS}$} & Liu, Youden & 5 & $46 \%(24,68)$ & $74 \%(68,79)$ \\
\hline & & Nearest $(0,1)$ & 6 & $55 \%(32,76)$ & $60 \%(54,66)$ \\
\hline & & Current literature & 8 & $82 \%(60,95)$ & $36 \%(30,42)$ \\
\hline & \multirow[t]{3}{*}{$H G+A L M / h^{2}+T U G$} & Liu, Nearest $(0,1)$ & 5 & $67 \%(35,90)$ & $74 \%(68,79)$ \\
\hline & & Youden & 4 & $58 \%(28,85)$ & $84 \%(79,88)$ \\
\hline & & Current literature & 8 & $92 \%(62,100)$ & $36 \%(30,41)$ \\
\hline & \multirow[t]{4}{*}{$\mathrm{HG}+\mathrm{ALM} / \mathrm{h}^{2}+\mathrm{SPPB}$} & Liu, & 7 & $84 \%(60,97)$ & $46 \%(40,52)$ \\
\hline & & Youden & 8 & $100 \%(82,100)$ & $37 \%(31,43)$ \\
\hline & & Nearest $(0,1)$ & 6 & $63 \%(38,84)$ & $60 \%(54,66)$ \\
\hline & & Current literature & 8 & $100 \%(82,100)$ & $37 \%(31,43)$ \\
\hline & \multirow[t]{3}{*}{$\mathrm{STS}+\mathrm{ALM} / \mathrm{h}^{2}+\mathrm{GS}$} & Liu, Nearest $(0,1)$ & 6 & $65 \%(50,79)$ & $63 \%(57,69)$ \\
\hline & & Youden & 8 & $94 \%(82,99)$ & $40 \%(33,46)$ \\
\hline & & Current literature & 8 & $94 \%(82,99)$ & $40 \%(33,46)$ \\
\hline & \multirow[t]{2}{*}{$\mathrm{STS}+\mathrm{ALM} / \mathrm{h}^{2}+\mathrm{TUG}$} & All & 5 & $67 \%(45,84)$ & $76 \%(70,81)$ \\
\hline & & Current literature & 8 & $96 \%(79,100)$ & $37 \%(31,43)$ \\
\hline & \multirow[t]{3}{*}{$\mathrm{STS}+\mathrm{ALM} / \mathrm{h}^{2}+\mathrm{SPPB}$} & Liu, Youden & 8 & $100 \%(93,100)$ & $41 \%(35,47)$ \\
\hline & & Nearest $(0,1)$ & 6 & $64 \%(49,77)$ & $63 \%(57,69)$ \\
\hline & & Current literature & 8 & $100 \%(93,100)$ & $41 \%(35,47)$ \\
\hline
\end{tabular}

$A L M / h^{2}$ Appendicular Lean Mass corrected for height, GS Gait speed, HG Handgrip strength, SPPB Short Physical Performance Battery, TUG Timed up and go

Highly sensitive tests have been proposed as ideal tools for screening purposes, allowing for the early identification and initiation of treatment [29]. Meanwhile, high specificity has been reported as the key diagnostic factor for a disease, with a combination of high sensitivity/low specificity and low sensitivity/high specificity test an ideal method to address false positives [29]. Using this model, the SPPB displays high sensitivity and represents the ideal screening tool for sarcopenia. Alternately, the SARC-F tool has consistently shown high specificity in numerous studies [30,31] and may therefore facilitate the diagnostic process. This combined approach represents an area for further research given the potential to enhance clinical care.

Sarcopenia in its current form includes the assessment of muscle strength, mass, and physical performance. However, whilst measures of strength and physical performance have shown links to adverse outcomes of sarcopenia, the same relationship is not observed with ALM [32, 33]. Our findings corroborate previous studies which have shown the disconnect between measures of muscle strength and physical performance and muscle mass. This is represented by the poor to acceptable AUC when using the SPPB as an independent diagnostic tool. However, a combination of SPPB with ALM $/ \mathrm{h}^{2}$ resulted in increased AUC, matching well with both initial and revised EWGSOP sarcopenia criteria, with the highest diagnostic value evident using the sit to stand test combined with $\mathrm{ALM} / \mathrm{h}^{2}$ and either gait speed or SPPB. This was somewhat expected given both the sit to stand test and gait speed are already components of the SPPB. However, it was surprising to observe that the combination of SPPB and ALM $/ \mathrm{h}^{2}$ yielded an increased AUC for all the other diagnostic criteria proposed by EWGSOP2. Given the SPPB is a tool which can be easily implemented into the routine care of patients without a need for specialized equipment, this finding may provide useful in clinical practice.

In the current study, we diagnosed participants presenting with severe sarcopenia which is a strength given that our previous findings showed that the severity of sarcopenia was the driving factor that increased falls and fractures [34]. Studies assessing the impact of sarcopenia in modulating balance performance are required to further efforts in developing interventions. The findings of the present study should also be taken in context of the limitations. Firstly, our population, although community-dwelling, reported a history of falls and/or fractures. Therefore, results may have been biased given that these participants are at increased risk for sarcopenia. Secondly, the cross-sectional 
nature of our study limits our findings as we are unable to determine whether longitudinally the participants classified as sarcopenic experienced adverse outcomes such as falls. Employing a longitudinal study design would have strengthened the findings.

\section{Conclusion}

This study provides an insight into the use of the SPPB for diagnosing sarcopenia according to the initial and revised EWGSOP definitions. Despite the acceptable diagnostic value, no cut-points could be determined which provided high sensitivity and specificity. However, a cutpoint of $\leq 8$ in the SPPB showed high sensitivity. Therefore, the SPPB may be used as a screening tool for sarcopenia to trigger further investigations and early intervention. The combination of the highly sensitive SPPB with a highly specific test may prove to be of great use to clinicians for diagnosing and treating sarcopenia, however, requires further investigation in longitudinal trials.

\section{Abbreviations}

EWGSOP: European Working Group on Sarcopenia in Older People; SPPB: Short Physical Performance Battery; DXA: Dual X-ray Absorptiometry; ALM $/ h^{2}$ : Appendicular Lean Mass corrected for Height squared; TUG: Timed Up and Go; AUC: Area Under receiver operating Curve

\section{Acknowledgements}

The authors would like to thank the Australian Institute for Musculoskeletal Science (AIMSS) and Melbourne Medical School for providing support to this Study.

\section{Authors' contributions}

GD conceived the study. SP, EBH, BK, SB and GD collected the data. SV performed statistical analysis. JZ and BK were major contributors to the writing of the manuscript. All authors read and approved the final manuscript.

\section{Funding}

This work was supported by the Australian Institute for Musculoskeletal Science (AIMSS). The sponsor did not play any role in in the design of the study, the collection, analysis, and interpretation of data or in writing the manuscript.

\section{Availability of data and materials}

The datasets used and/or analyzed during the current study are available from the corresponding author on reasonable request.

\section{Ethics approval and consent to participate}

This study was approved by the Western Health local Human Ethics Research Committee (DB2017.13 and QA2018.80-46205). Informed consent was waived as data was retrospectively collected from assessments performed during standard clinical care.

\section{Consent for publication}

Not applicable.

\section{Competing interests}

The authors declare that they have no competing interests.

\section{Author details}

${ }^{1}$ Australian Institute for Musculoskeletal Science (AIMSS), The University of Melbourne and Western Health, Sunshine Hospital, 176 Furlong Road, St Albans, VIC 3021, Australia. ²Department of Medicine-Western Health, The
University of Melbourne, 176 Furlong Road, St. Albans 3021, VIC, Australia. ${ }^{3}$ Subacute and Aged Care Services, Western Health, St. Albans, VIC, Australia.

Received: 20 February 2020 Accepted: 7 July 2020

Published online: 13 July 2020

References

1. Rizzoli R, Reginster JY, Arnal JF, Bautmans I, Beaudart C, Bischoff-Ferrari H, et al. Quality of life in sarcopenia and frailty. Calcif Tissue Int. 2013;93(2):10120.

2. Anker SD, Morley JE, von Haehling S. Welcome to the ICD-10 code for sarcopenia. J Cachexia Sarcopenia Muscle. 2016;7(5):512-4.

3. Cruz-Jentoft AJ, Baeyens JP, Bauer JM, Boirie Y, Cederholm T, Landi F, et al. Sarcopenia: European consensus on definition and diagnosis: report of the European working group on sarcopenia in older people. Age Ageing. 2010; 39(4):412-23.

4. Cruz-Jentoft AJ, Bahat G, Bauer J, Boirie Y, Bruyere O, Cederholm T, et al. Sarcopenia: revised European consensus on definition and diagnosis. Age Ageing. 2019;48(1):16-31.

5. Bhasin S, Travison TG, Manini TM, et al. Sarcopenia definition: the position statements of the sarcopenia definition and outcomes consortium [published online ahead of print, 2020 Mar 9]. J Am Geriatr Soc. 2020. https://doi.org/10.1111/jgs.16372.

6. Fielding RA, Vellas B, Evans WJ, Bhasin S, Morley JE, Newman AB, et al. Sarcopenia: an undiagnosed condition in older adults. Current consensus definition: prevalence, etiology, and consequences. International working group on sarcopenia. J Am Med Dir Assoc. 2011;12(4):249-56.

7. Chen LK, Liu LK, Woo J, Assantachai P, Auyeung TW, Bahyah KS, et al. Sarcopenia in Asia: consensus report of the Asian working Group for Sarcopenia. J Am Med Dir Assoc. 2014;15(2):95-101.

8. Malmstrom TK, Miller DK, Simonsick EM, Ferrucci L, Morley JE. SARC-F: a symptom score to predict persons with sarcopenia at risk for poor functional outcomes. J Cachexia Sarcopenia Muscle. 2016;7(1):28-36.

9. Phu S, Vogrin S, Zanker J, Bani Hassan E, Al Saedi A, Duque G. Agreement between initial and revised European working group on sarcopenia in older people definitions. people definitions. J Am Med Dir Assoc. 2019;20(3):3823.e1.

10. Guralnik JM, Simonsick EM, Ferrucci L, Glynn RJ, Berkman LF, Blazer DG, et al. A short physical performance battery assessing lower extremity function: association with self-reported disability and prediction of mortality and nursing home admission. J Gerontol. 1994:49(2):M85-94.

11. Yardley L, Beyer N, Hauer K, Kempen G, Piot-Ziegler C, Todd C. Development and initial validation of the falls efficacy scale-international (FES-I). Age Ageing. 2005;34(6):614-9.

12. Podsiadlo D, Richardson S. The timed "up \& go": a test of basic functional mobility for frail elderly persons. J Am Geriatr Soc. 1991;39(2):142-8.

13. Mandrekar JN. Receiver operating characteristic curve in diagnostic test assessment. J Thorac Oncol. 2010;5(9):1315-6.

14. Liu X. Classification accuracy and cut point selection. Stat Med. 2012;31(23): 2676-86.

15. Youden WJ. Index for rating diagnostic tests. Cancer. 1950;3(1):32-5

16. Fluss $R$, Faraggi $D$, Reiser $B$. Estimation of the Youden index and its associated cutoff point. Biom J. 2005:47(4):458-72.

17. Fisher S, Ottenbacher KJ, Goodwin JS, Graham JE, Ostir GV. Short physical performance battery in hospitalized older adults. Aging Clin Exp Res. 2009; 21(6):445-52.

18. Stookey AD, Katzel LI, Steinbrenner G, Shaughnessy M, Ivey FM. The short physical performance battery as a predictor of functional capacity after stroke. J Stroke Cerebrovasc Dis. 2014;23(1):130-5.

19. Bernabeu-Mora R, Gimenez-Gimenez LM, Montilla-Herrador J, GarciaGuillamon G, Garcia-Vidal JA, Medina-Mirapeix F. Determinants of each domain of the short physical performance battery in COPD. Int J Chron Obstruct Pulmon Dis. 2017:12:2539-44.

20. Owusu C, Margevicius S, Schluchter M, Koroukian SM, Berger NA. Short physical performance battery, usual gait speed, grip strength and vulnerable elders survey each predict functional decline among older women with breast cancer. J Geriatr Oncol. 2017:8(5):356-62.

21. Zhang F, Ferrucci L, Culham E, Metter EJ, Guralnik J, Deshpande N. Performance on five times sit-to-stand task as a predictor of subsequent falls and disability in older persons. J Aging Health. 2013; 25(3):478-92. 
22. Buatois S, Perret-Guillaume C, Gueguen R, Miget P, Vancon G, Perrin P, et al. A simple clinical scale to stratify risk of recurrent falls in communitydwelling adults aged 65 years and older. Phys Ther. 2010;90(4):550-60.

23. Fritz S, Lusardi M. White paper: "walking speed: the sixth vital sign". J Geriatr Phys Ther. 2009;32(2):46-9.

24. Perera S, Mody SH, Woodman RC, Studenski SA. Meaningful change and responsiveness in common physical performance measures in older adults. J Am Geriatr Soc. 2006;54(5):743-9.

25. Liguori I, Russo G, Aran L, Bulli G, Curcio F. Della-Morte, et al. sarcopenia: assessment of disease burden and strategies to improve outcomes. Clin Interv Aging. 2018;13:913-27.

26. Tieland $M$, Verdijk $L B$, de Groot $L C$, van Loon $L$. Handgrip strength does not represent an appropriate measure to evaluate changes in muscle strength during an exercise intervention program in frail older people. Int J Sport Nutr Exerc Metab. 2015;25(1):27-36.

27. Veronese N, Bolzetta F, Toffanello ED, Zambon S, De Rui M, Perissinotto E, et al. Association between short physical performance battery and falls in older people: the Progetto Veneto Anziani study. Rejuvenation Res. 2014; 17(3):276-84.

28. Landi F, Liperoti R, Russo A, Giovannini S, Tosato M, Capoluongo E, et al. Sarcopenia as a risk factor for falls in elderly individuals: results from the ilSIRENTE study. Clin Nutr. 2012;31(5):652-8.

29. Lalkhen AG, McCluskey A. Clinical tests: sensitivity and specificity. Contin Educ Anaesth Crit Care Pain. 2008:8(6):221-3.

30. Woo J, Leung J, Morley JE. Validating the SARC-F: a suitable community screening tool for sarcopenia? J Am Med Dir Assoc. 2014;15(9):630-4.

31. Barbosa-Silva TG, Menezes AM, Bielemann RM, Malmstrom TK, Gonzalez MC. Grupo de Estudos em Composicao corporal e N. enhancing SARC-F: improving sarcopenia screening in the clinical practice. J Am Med Dir Assoc. 2016;17(12):1136-41.

32. Schaap LA, Koster A, Visser M. Adiposity, muscle mass, and muscle strength in relation to functional decline in older persons. Epidemiol Rev. 2013;35: 51-65.

33. Kim YH, Kim Kl, Paik NJ, Kim KW, Jang HC, Lim JY. Muscle strength: a better index of low physical performance than muscle mass in older adults. Geriatr Gerontol Int. 2016;16(5):577-85

34. Sepulveda-Loyola W, Phu S, Bani Hassan E, Brennan-Olsen SL, Zanker J, Vogrin S, et al. The Joint Occurrence of Osteoporosis and Sarcopenia (Osteosarcopenia): Definitions and Characteristics. J Am Med Dir Assoc. 2020;21(2):220-5.

\section{Publisher's Note}

Springer Nature remains neutral with regard to jurisdictional claims in published maps and institutional affiliations.

Ready to submit your research? Choose BMC and benefit from:

- fast, convenient online submission

- thorough peer review by experienced researchers in your field

- rapid publication on acceptance

- support for research data, including large and complex data types

- gold Open Access which fosters wider collaboration and increased citations

- maximum visibility for your research: over $100 \mathrm{M}$ website views per year

At $\mathrm{BMC}$, research is always in progress.

Learn more biomedcentral.com/submissions 